\title{
Disease characteristics in patients with juvenile- and adult-onset systemic lupus erythematosus: A multi-center comparative study
}

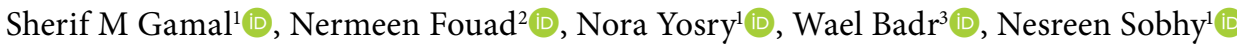 \\ ${ }^{1}$ Rheumatology, Faculty of Medicine, Cairo University, Cairo, Egypt \\ ${ }^{2}$ Rheumatology, Faculty of Medicine, Fayoum University, Fayoum, Egypt \\ ${ }^{3}$ Department of Pediatric, Faculty of Medicine, Fayoum University, Fayoum, Egypt
}

\begin{abstract}
Objectives: This study aims to compare disease characteristics in patients with juvenile-onset systemic lupus erythematosus (JSLE) and adult-onset systemic lupus erythematosus (ASLE).

Patients and methods: Between June 2010 and March 2020, a total of 186 patients with JSLE (23 males, 163 females; median age: 25 years; range, 20 to 30.3 years) and 236 patients with ASLE (23 males, 213 females; median age: 35 years; range, 29 to 40 years) were retrospectively analyzed. Clinical and laboratory data, treatment received, Systemic Lupus Erythematosus Disease Activity Index (SLEDAI) and Systemic Lupus International Collaborating Clinics (SLICC)/ACR Damage Index (SDI) scores, comorbidities and deaths were compared between the groups.

Results: The JSLE patients showed statistically significant higher constitutional manifestations, cardiac manifestations, serositis, nephritis, end-stage renal disease, neurological manifestations, gastrointestinal manifestations, secondary vasculitis, Raynaud's, livedo-reticularis, dry mouth, dry eye, ocular manifestations, avascular necrosis, hematological manifestations, and hypocomplementemia ( $p<0.001, p=0.016, p=0.005$, $\mathrm{p}=0.001, \mathrm{p}=0.04, \mathrm{p}<0.001, \mathrm{p}<0.001, \mathrm{p}<0.001, \mathrm{p}=0.002, \mathrm{p}=0.043, \mathrm{p}=0.004, \mathrm{p}=0.03, \mathrm{p}<0.001, \mathrm{p}=0.01, \mathrm{p}<0.001$, and $\mathrm{p}=0.001$, respectively). Median SLEDAI scores were statistically significant higher in the JSLE group, both at onset $(p<0.001)$ and in the final follow-up visit $(p<0.001)$. Median SLICC scores were also higher in the JSLE group $(p<0.001)$. Mycophenolate mofetil and intravenous pulse steroids were more frequently used in the juvenile group ( $<0.001$ and $p=0.03$, respectively). Hypertension, dyslipidemia, and avascular necrosis were found to be statistically significantly higher in the JSLE group ( $p<0.001, p=0.006$, and $p=0.01$, respectively). The mortality rate was statistically significantly higher in the JSLE group than the ASLE group $(p<0.001)$.

Conclusion: The JSLE patients showed more serious manifestations, higher disease activity, higher damage index, and mortality rate compared to ASLE patients. These results suggest the need of a regular follow-up and close surveillance of JSLE patients.

Keywords: Adult-onset, juvenile-onset, surveillance, systemic lupus erythematosus.
\end{abstract}

Systemic lupus erythematosus (SLE) is a multifactorial, multisystem autoimmune disease with extremely varied clinical presentations and fluctuating disease course, ranging from mild with gradual onset to rapidly fulminant multi-organ failure. ${ }^{1,2}$

Juvenile-onset SLE (JSLE) is one of the most common childhood autoimmune diseases; approximately 20\% of SLE cases are diagnosed in childhood and adolescence, usually at approximately 11 to 12 years of age..$^{3,4}$ It demarcates a subset of individuals that fulfill the classification criteria for SLE in children younger than 16 years. ${ }^{5-7}$

Previous studies have proposed that the age of disease onset may affect disease expression,

Received: April 12, 2021 Accepted: June 27, 2021 Published online: December 24, 2021

Correspondence: Nesreen Sobhy, MD. Rheumatology, Faculty of Medicine, Cairo University, 11562 Cairo, Egypt. Tel: 01002982012 e-mail: nesreen_sobhy@yahoo.com

Citation:

Gamal SM, Fouad N, Yosry N, Badr W, Sobhy N. Disease characteristics in patients with juvenile- and adult-onset systemic lupus erythematosus: A multi-center comparative study. Arch Rheumatol 2022;37(2):280-287. 
serological findings, and the pattern of organ involvement. ${ }^{8,9}$ In general, JSLE is acute, with higher disease activity and severity, and with more permanent damage compared to adult-onset SLE (ASLE). ${ }^{8}$

In most studies comparing JSLE and ASLE, nephritis has been reported to be more common in JSLE patients, ${ }^{10-12}$ and mucocutaneous manifestations, neuropsychiatric manifestations, and cytopenia are more frequently reported in JSLE. ${ }^{11,12}$ Torrente-Segarra et al. ${ }^{12}$ found that JSLE showed significantly more pleuritis, pericarditis, Raynaud's phenomenon, hypocomplementemia, anti-double-stranded deoxyribonucleic acid (dsDNA), while anti-Ro antibodies, secondary Sjögren syndrome, osteoporosis and fibromyalgia were more common in ASLE. Furthermore, Smith et al. $^{13}$ reported that cutaneous vasculitis was more prevalent in JSLE compared to adult-onset disease.

Although survival rates for patients with JSLE have significantly improved in recent years, morbidity is still high, and most adolescents and young adults with JSLE continue to have an active disease. These patients are still developing new organ involvement and damage ${ }^{14,15}$ with subsequent impaired health-related quality of life. ${ }^{16}$

It is well known that ethnicity and sociodemographic factors affect the characteristics of JSLE. ${ }^{17}$ Although JSLE is not uncommon, ${ }^{18}$ only a limited number of studies discuss the long-term outcomes of JSLE. In the present study, we aimed to investigate the clinical characteristics, disease activity, and damage among patients with JSLE and compare these clinical characteristics with ASLE patients. Such a characterization may help to raise awareness about this disease category, allowing better understanding of the disease pattern, outcomes and prognosis.

\section{PATIENTS AND METHODS}

This multi-center, retrospective, comparative study was conducted at Rheumatology Department in Cairo and Fayoum University, and in Pediatric Department in Fayoum University between June 2010 and March
2020. Medical records of a total of 186 patients with JSLE (23 males, 163 females; median age: 25 years; range, 20 to 30.3 years) and 236 patients with ASLE (23 males, 213 females; median age: 35 years; range, 29 to 40 years) were included. The ASLE patients consisted the control group and were randomly selected. All patients and controls fulfilled the 1987 American College of Rheumatology (ACR) classification criteria for SLE. ${ }^{7}$

The medical records of all patients were reviewed for their demographic, clinical, laboratory, and immunological data, as well as treatment received, complications, associated comorbidities, outcomes, and deaths. Disease activity both at onset and during the last visit according to the Systemic Lupus Erythematosus Disease Activity Index (SLEDAI). ${ }^{19}$ and Systemic Lupus International Collaborating Clinics (SLICC)/ACR Damage Index (SDI) were reported. ${ }^{20}$

\section{Statistical analysis}

Statistical analysis was performed using the SPSS for Windows version 15.0 software (SPSS Inc., Chicago, IL, USA). Descriptive data were expressed in median (min-max) or number and frequency. The Kolmogorov-Smirnov test was used for normality testing. Comparisons between the study groups were done using the Mann-Whitney test for quantitative variables and the chi-square test for the qualitative variables. Odds ratios (ORs) were calculated with 95\% confidence interval (CI). A two-tailed $p$ value of $<0.05$ was considered statistically significant.

\section{RESULTS}

Of a total of 422 patients with SLE, 186 (44.1\%) of them were classified as JSLE (age at onset $\leq 16$ years) and 236 were classified as ASLE (age at onset $>16$ years). The median age of onset was 14 (range, 12 to 15) years and the median disease duration was 12 (range, 6.75 to 17.25 ) years in the JSLE group. The median age of onset was 25 (range, 21 to $32.75)$ years and the median disease duration was six (range, 3.25 to 11 ) years in the ASLE group. There was no significant difference in the sex distribution between the JSLE and ASLE groups $(p=0.4)$. 
Patients with JSLE showed significantly higher constitutional manifestations, cardiac manifestations, serositis, nephritis, end-stage renal disease, neurological manifestations, gastrointestinal (GI) manifestations, secondary vasculitis, Raynaud's phenomenon, livedo-reticularis, intra-alveolar hemorrhage, secondary sicca (dry mouth and dry eyes), and ocular manifestations compared to the ASLE group. Clinical manifestations are illustrated in Table 1.

Disease activity scores as measured by the SLEDAI were significantly higher in the juvenile group than in the adult group, both at baseline (11 [6 to 17.25 ] vs. 8 [4 to 13], respectively; $\mathrm{p}<0.001)$ and at the final visit $(4[0$ to 8$]$ vs. 0 [0 to 4], respectively; $p<0.001$ ). Similarly, SDIs measured by the SLICC scores were also higher in the juvenile group ( 1 [0 to 2$)$ vs. 0 [0 to 1$]$, respectively; $\mathrm{p}<0.001)$.

Hematological manifestations $(p<0.001)$ and hypocomplementemia $(p=0.001)$ were significantly more frequent in the JSLE group. Laboratory test results are shown in Table 2.
The median steroid dose given during the last visit was 15 (range, 9.3 to 25) $\mathrm{mg}$ in patients with JSLE and 10 (range, 5 to 20) $\mathrm{mg}$ in patients with ASLE.

Mycophenolate mofetil (MMF) $(\mathrm{p}<0.001)$ and intravenous (IV) pulse steroids $(p=0.034)$ were more frequently used in JSLE. Otherwise, no significant differences were found in the other treatment options (Table 3).

Hypertension, dyslipidemia and avascular necrosis were found to be significantly higher in the patients with JSLE than ASLE $(p<0.001$, $p=0.006$, and $p=0.01$, respectively) (Table 4).

In addition, JSLE patients showed a statistically significant higher mortality rate compared to ASLE patients (34/186, 18.3\% vs. $13 / 236,5.5 \%$, respectively; $p<0.001$ ), with infection in 6/34 (17.6\%) and single organ failure (e.g., renal failure) in 6/34 (17.6\%) being the most reported causes of deaths in the JSLE patients. Other causes of death included alveolar hemorrhage in 3/34 (8.8\%), bleeding peptic ulcer in $1 / 34$ (2.9\%), and

Table 1. Clinical manifestations of the participants

\begin{tabular}{|c|c|c|c|c|c|}
\hline & \multicolumn{2}{|c|}{ Juvenile-onset lupus ( $\mathrm{n}=186$ ) } & \multicolumn{2}{|c|}{ Adult-onset lupus ( $\mathrm{n}=236$ ) } & \multirow[b]{2}{*}{$p$} \\
\hline & $\mathrm{n}$ & $\%$ & $\mathrm{n}$ & $\%$ & \\
\hline Constitutional manifestations & 153 & 82.3 & 158 & 66.9 & $<0.001$ \\
\hline Mucocutaneous manifestations & 164 & 88.2 & 194 & 82.2 & 0.09 \\
\hline Pulmonary manifestations & 107 & 57.5 & 117 & 49.6 & 0.104 \\
\hline Cardiac manifestations & 54 & 29.0 & 45 & 19.1 & 0.016 \\
\hline Serositis & 106 & 57.0 & 102 & 43.2 & 0.005 \\
\hline Nephritis & 140 & 75.3 & 143 & 60.6 & 0.001 \\
\hline End stage renal disease & 17 & 9.1 & 10 & 4.2 & 0.04 \\
\hline Neurological manifestations & 87 & 46.8 & 70 & 29.7 & $<0.001$ \\
\hline Gastrointestinal manifestations & 48 & 25.8 & 23 & 9.7 & $<0.001$ \\
\hline Musculoskeletal manifestations & 164 & 88.2 & 209 & 88.6 & 0.902 \\
\hline Secondary vasculitis & 71 & 38.1 & 54 & 22.8 & $<0.001$ \\
\hline Thrombosis & 22 & 11.8 & 43 & 18.2 & 0.07 \\
\hline Raynaud's phenomenon & 51 & 27.4 & 36 & 15.3 & 0.002 \\
\hline Livedo-reticularis & 11 & 5.9 & 5 & 2.1 & 0.043 \\
\hline Digital gangrene & 7 & 3.8 & 5 & 2.1 & 0.3 \\
\hline Intra-alveolar hemorrhage & 5 & 2.7 & 1 & 0.4 & 0.092 \\
\hline Dry mouth & 15 & 8.1 & 5 & 2.1 & 0.004 \\
\hline Dry eye & 11 & 5.9 & 4 & 1.7 & 0.03 \\
\hline Ocular manifestations & 27 & 14.5 & 10 & 4.2 & $<0.001$ \\
\hline Pulmonary hypertension & 24 & 12.9 & 20 & 8.5 & 0.14 \\
\hline
\end{tabular}


Tabli 2. Laboratory data of the participants

\begin{tabular}{|c|c|c|c|c|c|}
\hline & \multicolumn{2}{|c|}{ Juvenile-onset lupus ( $\mathrm{n}=186$ ) } & \multicolumn{2}{|c|}{ Adult-onset lupus ( $\mathrm{n}=236$ ) } & \multirow[b]{2}{*}{$p$} \\
\hline & $\mathrm{n}$ & $\%$ & $\mathrm{n}$ & $\%$ & \\
\hline Hematological manifestations & 176 & 94.6 & 190 & 80.5 & $<0.001$ \\
\hline Anemia throughout the disease & 168 & 90.3 & 187 & 79.2 & 0.002 \\
\hline Leukopenia throughout & 96 & 51.6 & 93 & 39.4 & 0.012 \\
\hline Thrombocytopenia throughout & 79 & 42.5 & 46 & 19.5 & $<0.001$ \\
\hline ANA positivity & $177 / 182$ & 97.3 & $228 / 235$ & 97 & 0.89 \\
\hline Anti-dsDNA antibodies positivity & $116 / 154$ & 75.3 & $156 / 229$ & 68.1 & 0.1 \\
\hline Hypocomplementemia & $63 / 158$ & 39.9 & $54 / 223$ & 24.2 & 0.001 \\
\hline APL antibody positivity & $58 / 131$ & 44.3 & $65 / 183$ & 35.5 & 0.1 \\
\hline
\end{tabular}

Table 3. Treatment options given to participants

\begin{tabular}{|c|c|c|c|c|c|}
\hline & \multicolumn{2}{|c|}{ Juvenile-onset lupus ( $n=186$ ) } & \multicolumn{2}{|c|}{ Adult-onset lupus ( $\mathrm{n}=236$ ) } & \multirow[b]{2}{*}{$p$} \\
\hline & $\mathrm{n}$ & $\%$ & $\mathrm{n}$ & $\%$ & \\
\hline Cyclophosphamide & 105 & 56.5 & 115 & 48.7 & 0.11 \\
\hline Azathioprine & 147 & 79.0 & 168 & 71.2 & 0.066 \\
\hline Antimalarial & 176 & 94.6 & 223 & 94.5 & 0.95 \\
\hline MMF & 77 & 41.4 & 45 & 19.1 & $<0.001$ \\
\hline IV pulse steroids & 147 & 79.0 & 165 & 69.9 & 0.034 \\
\hline
\end{tabular}

Table 4. Associated comorbidities of participants

\begin{tabular}{|c|c|c|c|c|c|}
\hline & \multicolumn{2}{|c|}{ Juvenile-onset lupus ( $\mathrm{n}=186$ ) } & \multicolumn{2}{|c|}{ Adult-onset lupus ( $\mathrm{n}=236)$} & \multirow[b]{2}{*}{$p$} \\
\hline & $\mathrm{n}$ & $\%$ & $\mathrm{n}$ & $\%$ & \\
\hline Hypertension & 82 & 44.1 & 64 & 27.1 & $<0.001$ \\
\hline Diabetes mellitus & 12 & 6.5 & 19 & 8.1 & 0.5 \\
\hline Dyslipidemia & 75 & 40.3 & 65 & 27.5 & 0.006 \\
\hline Thyroid dysfunction & 10 & 5.4 & 7 & 3.0 & 0.21 \\
\hline Malignancy & 1 & 0.5 & 0 & 0 & 0.44 \\
\hline Osteoporosis & $27 / 150$ & 18 & 34 & 14.4 & 0.3 \\
\hline Avascular necrosis & 23 & 12.4 & 13 & 5.5 & 0.01 \\
\hline
\end{tabular}

pulmonary edema in 1/34 (2.9\%). Combination of (single organ failure and infection), (alveolar hemorrhage and infection), and (organ failure with infection and alveolar hemorrhage) were recorded as the cause of death in $3 / 34$ (8.8\%). In $14 / 34(41.2 \%)$ of the JSLE patients, the exact cause of death could not be identified due to either home death or missing data in the medical records.

\section{DISCUSSION}

In this study, we assessed the clinical, immunological, and serological characteristics 
of 186 patients with JSLE and compared these findings with those of 236 patients with ASLE. Although many studies have addressed the clinical and immunological features of JSLE, only a few comparisons between JSLE and ASLE have been made, and most of which have conflicting results.

Our study showed that the female-to-male ratio was 7:1 and 9:1 in JSLE and ASLE, respectively. The sex ratio of ASLE was similar to previous studies ${ }^{21,22}$ while that of JSLE was lower than the previous reports. ${ }^{23}$ In a study by Gheita et al., ${ }^{24}$ the female-to-male ratio was 6.7:1 and 10.5:1 for JSLE and ASLE, respectively, consistent with our findings. However, a lower female-to-male ratio in JSLE was reported in other studies from Saudi Arabia $^{25}$ and Kuwait. ${ }^{26}$

While comparing the prevalence of clinical manifestations between JSLE and ASLE, we found that JSLE showed significantly higher rates of constitutional, cardiac, neurological and GI manifestations. These patients were also more likely to develop serositis, nephritis, end-stage renal disease, and secondary vasculitis compared to their adult counterparts. Mucocutaneous and musculoskeletal manifestations are the most common clinical manifestations in JSLE, whereas musculoskeletal manifestations are the most common clinical manifestations in ASLE. Our findings are similar to previous reports. ${ }^{25-28}$

There are variations between the studies regarding the prevalence of various clinical manifestations. In a study, there was no significant difference between ASLE and JSLE regarding serositis and constitutional, mucocutaneous, renal, and vascular manifestations. ${ }^{29}$ Another study from Egypt showed that nephritis and neuropsychiatric and hematological manifestations occurred more frequently in children. ${ }^{27}$ Similar findings were also described previously. ${ }^{30-32}$ In a large-scale Turkish study, nephritis, neurological, mucocutaneous and hematological manifestations were more frequent in JSLE patients. ${ }^{11}$ Similar findings were reported in das Chagas Medeiros et al.'s, ${ }^{10}$ Torrente-Segarra et al.'s, ${ }^{12}$ and Brunner et al.'s ${ }^{16}$ studies. This variability is mostly due to different sample sizes and selection criteria including different age groups among JSLE. Other causes may be race, ethnicity, and environmental and developmental factors.
In the present study, the SLEDAI scores were significantly higher in patients with JSLE than in patients with ASLE. This finding is consistent with previous studies. ${ }^{27,29,33,34}$ Systemic lupus erythematosus-related major organ involvement such as nephritis and neuropsychiatric manifestations may be the main cause of the increased SLEDAI scores. ${ }^{27}$ Moreover, adults seem to respond better to treatment and are able to achieve and maintain remission in a shorter period compared to juveniles. ${ }^{30}$ It may also highlight the possibility that the current medications available for ASLE may not work as efficiently in JSLE.

Similarly, the SLICC damage scores were also higher in the patients with JSLE in our study, consistent with a previous study. ${ }^{24}$ The increased scores in JSLE were mainly attributed to renal and musculoskeletal damage. Moreover, regardless of the damage index used, damage in JSLE is always higher than that in ASLE. In a study by Brunner et al., ${ }^{30}$ the SLICC/ACR SDI was used as to measure disease damage. Although SDI is more directly related to aging (by assessing cataracts, myocardial infarction, and diabetes mellitus), patients with JSLE still had a higher disease damage index than patients with ASLE in our study. This can be explained by the increased disease activity and higher levels of corticosteroids and immunosuppressive agents required for disease control in children, compared to adults. ${ }^{30}$

Serological analysis showed that complement consumption was significantly more frequent in JSLE, whereas antinuclear antibody (ANA), anti-dsDNA, and anti-phospholipid (APL) antibodies showed no significant difference. A review of the current literature reveals some variations in serological abnormalities; complement consumption is a common finding in many studies. ${ }^{27,35}$ Other studies have shown that serum C3 is consumed without significant differences between the groups, whereas serum C4 was lower than normal in JSLE, but not in ASLE. ${ }^{3,29}$ The C3 is correlated with nephritis activity and, as patients with JSLE are more likely to develop active and progressive nephritis than patients with ASLE, C3 consumption levels are usually higher in this group. ${ }^{27}$ Similar to our results, previous studies found no significant differences between the levels of ANA and 
anti-dsDNA antibodies in both groups. ${ }^{3,10,36}$ On the other hand, some studies found that antidsDNA antibodies were more frequently detected in children. ${ }^{27,37}$ Regarding the APL antibodies, we had similar results with a previous study by Gyo et al. ${ }^{36}$ However, some other studies found higher APL antibodies in patients with JSLE. ${ }^{27,38}$

In the current study, MMF and IV pulse steroids were more frequently used in JSLE than in ASLE. Comparative studies between adults and children found that JSLE was more frequently treated with high doses of corticosteroids and immunosuppressive medications than ASLE. ${ }^{39}$ In a study by Brunner et al., ${ }^{30}$ children with SLE were treated with IV methylprednisolone almost three times more often than adults with SLE. This may be explained by the more active, poorly controlled disease in JSLE compared to ASLE.

In our study, hypertension, dyslipidemia, and avascular necrosis were significantly higher in JSLE than in ASLE. The association of these comorbidities with JSLE can be explained by the higher prevalence of nephritis in JSLE and, consequently, the higher need of IV pulse and oral steroid.

The association between corticosteroids and avascular necrosis has been shown in previous studies. ${ }^{40,41}$ Furthermore, hypertension and dyslipidemia are considered risk factors for avascular necrosis, ${ }^{40,41}$ which may explain the higher incidence of avascular necrosis among our JSLE patients.

In our study, JSLE was found to have significantly higher mortality rates compared to ASLE (18.3\% vs. $5.5 \%$, respectively; $p<0.001)$. Although most reports have shown a decline in mortality rates among JSLE, this is still higher than the general population. Mortality in JSLE is five times higher than in ASLE patients. The main causes of death in our pediatric patients were infection and organ failure, consistent with the literature. ${ }^{26,42,43}$ Since JSLE is associated with higher disease activity, damage indices, and comorbidities, it is unsurprising that JSLE has higher mortality rates with diverse causes of mortality.

The main strength of this study is the large number of patients with JSLE, recruited from two different centers. On the other hand, due to the retrospective nature of the study, potential limitations include the inability to retrieve some missing data as cumulative oral steroid dose for each patient, detailed causes of mortality in some patients, and anti-Ro antibody results.

In conclusion, our study findings provide a better understanding of the clinical characteristics, disease activity, long-term outcomes, and damage in JSLE patients. These results highlight the importance of close monitoring, follow-up, and adequate management of JSLE to minimize the increased morbidity and mortality of these young patients in the future.

Ethics Committee Approval: The study was approved by the participating department in Cairo and Fayoum University in January 2019. The study was conducted in accordance with the principles of the Declaration of Helsinki.

Data Sharing Statement: The data that support the findings of this study are available from the corresponding author upon reasonable request.

Author Contributions: All authors contributed equally to the article: S.M.G., N.F., N.Y., W.B., N.S.

Conflict of Interest: The authors declared no conflicts of interest with respect to the authorship and/or publication of this article.

Funding: The authors received no financial support for the research and/or authorship of this article.

\section{REFERENCES}

1. Teruel M, Alarcón-Riquelme ME. The genetic basis of systemic lupus erythematosus: What are the risk factors and what have we learned. J Autoimmun 2016;74:161-75.

2. Levy DM, Kamphuis S. Systemic lupus erythematosus in children and adolescents. Pediatr Clin North Am 2012;59:345-64.

3. Tarr T, Dérfalvi B, Győri N, Szántó A, Siminszky Z, Malik A, et al. Similarities and differences between pediatric and adult patients with systemic lupus erythematosus. Lupus 2015;24:796-803.

4. Klein-Gitelman M, Reiff A, Silverman ED. Systemic lupus erythematosus in childhood. Rheum Dis Clin North Am 2002;28:561-77.

5. Silva CA, Avcin T, Brunner HI. Taxonomy for systemic lupus erythematosus with onset before adulthood. Arthritis Care Res (Hoboken) 2012;64:1787-93.

6. Petri M, Orbai AM, Alarcón GS, Gordon C, Merrill JT, Fortin PR, et al. Derivation and validation of the 
Systemic Lupus International Collaborating Clinics classification criteria for systemic lupus erythematosus. Arthritis Rheum 2012;64:2677-86.

7. Hochberg MC. Updating the American College of Rheumatology revised criteria for the classification of systemic lupus erythematosus. Arthritis Rheum 1997;40:1725.

8. Descloux E, Durieu I, Cochat P, Vital-Durand D, Ninet J, Fabien $\mathrm{N}$, et al. Influence of age at disease onset in the outcome of paediatric systemic lupus erythematosus. Rheumatology (Oxford) 2009;48:779-84.

9. Kang JH, Park DJ, Lee KE, Lee JS, Choi YD, Lee SS. Comparison of clinical, serological, and prognostic differences among juvenile-, adult-, and late-onset lupus nephritis in Korean patients. Clin Rheumatol 2017;36:1289-95.

10. das Chagas Medeiros MM, Bezerra MC, Braga FN, da Justa Feijão MR, Gois AC, Rebouças VC, et al. Clinical and immunological aspects and outcome of a Brazilian cohort of 414 patients with systemic lupus erythematosus (SLE): Comparison between childhood-onset, adult-onset, and late-onset SLE. Lupus 2016;25:355-63.

11. Artim-Esen B, Şahin S, Çene E, Şahinkaya Y, Barut K, Adrovic A, et al. Comparison of disease characteristics, organ damage, and survival in patients with juvenile-onset and adult-onset systemic lupus erythematosus in a combined cohort from 2 tertiary centers in Turkey. J Rheumatol 2017;44:619-25.

12. Torrente-Segarra V, Salman Monte TC, Rúa-Figueroa I, Sánchez-Alonso F, López-Longo FJ, GalindoIzquierdo $\mathrm{M}$, et al. Juvenile- and adult-onset systemic lupus erythematosus: A comparative study in a large cohort from the Spanish Society of Rheumatology Lupus Registry (RELESSER). Clin Exp Rheumatol 2017;35:1047-55.

13. Smith EMD, Lythgoe H, Hedrich CM. Vasculitis in juvenile-onset systemic lupus erythematosus. Front Pediatr 2019;7:149.

14. Hersh AO, Trupin L, Yazdany J, Panopalis P, Julian L, Katz P, et al. Childhood-onset disease as a predictor of mortality in an adult cohort of patients with systemic lupus erythematosus. Arthritis Care Res (Hoboken) 2010;62:1152-9.

15. Lim LSH, Pullenayegum E, Feldman BM, Lim L, Gladman DD, Silverman ED. From childhood to adulthood: Disease activity trajectories in childhoodonset systemic lupus erythematosus. Arthritis Care Res (Hoboken) 2018;70:750-7.

16. Brunner HI, Higgins GC, Wiers K, Lapidus SK, Olson $\mathrm{JC}$, Onel K, et al. Health-related quality of life and its relationship to patient disease course in childhoodonset systemic lupus erythematosus. J Rheumatol 2009;36:1536-45.

17. Kurahara D, Tokuda A, Grandinetti A, Najita J, Ho $\mathrm{C}$, Yamamoto $\mathrm{K}$, et al. Ethnic differences in risk for pediatric rheumatic illness in a culturally diverse population. J Rheumatol 2002;29:379-83.
18. el-Garf A, Salah S. Juvenile systemic lupus erythematosus among Egyptian children. J Rheumatol 1990;17:1168-70.

19. Bombardier C, Gladman DD, Urowitz MB, Caron $\mathrm{D}$, Chang $\mathrm{CH}$. Derivation of the SLEDAI. A disease activity index for lupus patients. The Committee on Prognosis Studies in SLE. Arthritis Rheum 1992;35:630-40.

20. Gladman D, Ginzler E, Goldsmith C, Fortin P, Liang M, Urowitz $\mathrm{M}$, et al. The development and initial validation of the Systemic Lupus International Collaborating Clinics/American College of Rheumatology damage index for systemic lupus erythematosus. Arthritis Rheum 1996;39:363-9.

21. Khan D, Ansar Ahmed S. The immune system is a natural target for estrogen action: Opposing effects of estrogen in two prototypical autoimmune diseases. Front Immunol 2016;6:635.

22. Rees F, Doherty M, Grainge MJ, Lanyon P, Zhang W. The worldwide incidence and prevalence of systemic lupus erythematosus: A systematic review of epidemiological studies. Rheumatology (Oxford) 2017;56:1945-61.

23. Lewandowski LB, Schanberg LE, Thielman N, Phuti A, Kalla AA, Okpechi I, et al. Severe disease presentation and poor outcomes among pediatric systemic lupus erythematosus patients in South Africa. Lupus 2017;26:186-94.

24. Gheita TA, Fawzy SM, Nour El-din AM, El-Fishawyc HS. Juvenile and adult onset systemic lupus erythematosus outcome in Egyptian patients. Egypt Rheumatol 2011;33:99-105.

25. Bahabri S, Sabban EA, Al Rashed A, Al-Mayouf S, Al Mazyed A, Abdulrazik A, et al. Juvenile systemic lupus erythematosus in 60 Saudi children. Ann Saudi Med 1997;17:612-5.

26. Alsaeid K, Kamal H, Haider MZ, Al-Enezi HM, Malaviya AN. Systemic lupus erythematosus in Kuwaiti children: Organ system involvement and serological findings. Lupus 2004;13:613-7.

27. Mohamed DF, El-Din Abdel Aziz AB, AbdelMoteleb Hassan S, Shedid NH, El-Owaidy RH, El Moniem Teama MA. Juvenile lupus: Different clinical and serological presentations compared to adult lupus in Egypt. The Egyptian Rheumatologist 2018;40:55-8.

28. Cherif F, Mebazaa A, Mokni M, El Euch D, Azaiz MI, Dhahri AB. Childhood discoid lupus erythematosus: A Tunisian retrospective study of 16 cases. Pediatr Dermatol 2003;20:295-8.

29. Abdel-Nabi HH, Abdel-Noor RA. Comparison between disease onset patterns of Egyptian juvenile and adult systemic lupus erythematosus (single centre experience). Lupus 2018;27:1039-44.

30. Brunner HI, Gladman DD, Ibañez D, Urowitz MD, Silverman ED. Difference in disease features between childhood-onset and adult-onset systemic lupus erythematosus. Arthritis Rheum 2008;58:556-62. 
31. Tucker LB, Uribe AG, Fernández M, Vilá LM, McGwin $\mathrm{G}$, Apte $\mathrm{M}$, et al. Adolescent onset of lupus results in more aggressive disease and worse outcomes: Results of a nested matched case-control study within LUMINA, a multiethnic US cohort (LUMINA LVII). Lupus 2008;17:314-22.

32. Hoffman IE, Lauwerys BR, De Keyser F, Huizinga TW, Isenberg D, Cebecauer L, et al. Juvenile-onset systemic lupus erythematosus: Different clinical and serological pattern than adult-onset systemic lupus erythematosus. Ann Rheum Dis 2009;68:412-5.

33. Ramírez Gómez LA, Uribe Uribe O, Osio Uribe O, Grisales Romero H, Cardiel MH, Wojdyla D, et al. Childhood systemic lupus erythematosus in Latin America. The GLADEL experience in 230 children. Lupus 2008;17:596-604.

34. Balci S, Ekinci RMK, Bayazit AK, Melek E, Dogruel $\mathrm{D}$, Altintas DU, et al. Juvenile systemic lupus erythematosus: A single-center experience from southern Turkey. Clin Rheumatol 2019;38:145968.

35. Choi JH, Park DJ, Kang JH, Yim YR, Lee KE, Lee $\mathrm{JW}$, et al. Comparison of clinical and serological differences among juvenile-, adult-, and late-onset systemic lupus erythematosus in Korean patients. Lupus 2015;24:1342-9.

36. Gokce M, Bilginer Y, Besbas N, Ozaltin F, Cetin M, Gumruk F, et al. Hematological features of pediatric systemiclupuserythematosus: Suggesting management strategies in children. Lupus 2012;21:878-84.
37. Levy DM, Peschken CA, Tucker LB, Chédeville G, Huber AM, Pope JE, et al. Influence of ethnicity on childhood-onset systemic lupus erythematosus: Results from a multiethnic multicenter Canadian cohort. Arthritis Care Res (Hoboken) 2013;65:152-60.

38. Papadimitraki ED, Isenberg DA. Childhood- and adultonset lupus: An update of similarities and differences. Expert Rev Clin Immunol 2009;5:391-403.

39. Mina R, Brunner HI. Pediatric lupus--are there differences in presentation, genetics, response to therapy, and damage accrual compared with adult lupus? Rheum Dis Clin North Am 2010;36:53-80.

40. Tsai HL, Chang JW, Lu JH, Liu CS. Epidemiology and risk factors for avascular necrosis in childhood systemic lupus erythematosus in a Taiwanese population. Sci Rep 2020;10:15563.

41. Joo YB, Sung YK, Shim JS, Kim JH, Lee EK, Lee $\mathrm{HS}$, et al. Prevalence, incidence, and associated factors of avascular necrosis in Korean patients with systemic lupus erythematosus: A nationwide epidemiologic study. Rheumatol Int 2015;35:879-86.

42. Gheith RE, El-Gazzar II, El Fishawy HS, Nour El-Din AM, Bahgat DMR, Gheita TA. Juvenile and juvenileonset systemic lupus erythematosus patients: Clinical characteristics, disease activity and damage. Egyptian Pediatric Association Gazette 2017;65:49-53.

43. Tavangar-Rad F, Ziaee V, Moradinejad MH, Tahghighi F. Morbidity and mortality in Iranian children with juvenile systemic lupus erythematosus. Iran $\mathrm{J}$ Pediatr 2014;24:365-70. 